\title{
Impact of a Camp upon Disease-Specific Knowledge and Quality of Life in Children and Adolescents with Inflammatory Bowel Disease
}

\author{
Robert N. Lopez ${ }^{a} \quad$ Andrew McCombie ${ }^{b} \quad$ Richard B. Gearry ${ }^{b} \quad$ Andrew S. Day ${ }^{a}$ \\ ${ }^{a}$ Department of Paediatrics, University of Otago, Christchurch, New Zealand; b Department of Medicine, University \\ of Otago, Christchurch, New Zealand
}

\section{Keywords}

Children · Camp · Inflammatory bowel disease · Paediatric patient · Quality of life

\begin{abstract}
Children with inflammatory bowel disease (IBD) who attend residential camps derive various psycho-social benefits from their attendance. This study evaluated the impact of camp attendance on participants' disease-specific knowledge and quality of life (QOL). Prior to attending a dedicated camp for children with IBD, all campers were contacted and asked to participate in an evaluation of outcomes. Campers were asked to complete questionnaires regarding background disease status, demographic information, disease-specific knowledge (Inflammatory Bowel Disease Knowledge Inventory Device [IBD-KID]) and QOL (IMPACT-III). Assessments were completed before the camp and then again 1 month and 6 months after the camp. The camp consisted of adventure-based experiences and did not include specific IBD-related educational activities. Thirty-nine of 44 campers provided baseline information. The responders comprised 21 boys, and the median age was 14 years. Most $(n=35)$ were
\end{abstract}

diagnosed with Crohn's disease. Twenty-five of the baseline responders were in clinical remission. Mean IBD-KID scores increased from baseline at both 1 month and 6 months ( $p=$ 0.03 and $p=0.04$, respectively). Although mean $\mathrm{QOL}$ scores did not increase after 1 or 6 months, body image sub-scores were increased at 6 months $(p=0.015)$. Children and adolescents with IBD attending this residential camp demonstrated enhanced disease-specific knowledge following the camp, which was maintained 6 months following the camp. QOL scores were not increased overall at either time point. These results demonstrate a further benefit of residential camps for children and adolescents with IBD.

(c) 2020 The Author(s)
Published by S. Karger AG, Basel

\section{Introduction}

The incidence of inflammatory bowel disease (IBD) in children and adolescents is increasing across the globe [1]. High rates of IBD have been shown in New Zealand,

R.N.L. and A.M. contributed equally to this work.

\begin{tabular}{ll}
\hline KARGER & $\begin{array}{l}\text { ๑ } 2020 \text { The Author(s) } \\
\text { Published by S. Karger AG, Basel }\end{array}$ \\
karger@karger.com & Karger \\
This article is licensed under the Creative Commons Attribution- \\
www.karger.com/iid & $\begin{array}{l}\text { NonCommercial-NoDerivatives 4.0 International License (CC BY- } \\
\text { NC-ND) (http://www.karger.com/Services/OpenAccessLicense). } \\
\text { Usage and distribution for commercial purposes as well as any dis- } \\
\text { tribution of modified material requires written permission. }\end{array}$
\end{tabular}

Prof. Andrew S. Day

Department of Paediatrics, Cure Kids Chair of Paediatric Research University of Otago, PO Box 4345

Christchurch 8140 (New Zealand)

andrew.day@otago.ac.nz 
with increasing incidence documented [2, 3]. Many reports illustrate that IBD can impact adversely upon the psycho-social well-being of individuals [4].

Patients learn about their condition after diagnosis. The extent and quality of knowledge and understanding likely impacts adherence to medical therapies, disease outcome and quality of life (QOL) [5]. Adult and paediatric tools are available for the assessment of disease-specific knowledge [6, 7].

IBD-specific camps are proven to lead to beneficial effects upon patients who attend them. A previous report demonstrated that participant attendance at New Zealand's first IBD camp had a favourable impact upon patient confidence, acceptance of disease and ability to form friendships [8]. A previous exploratory study also showed that children with IBD had improved health-related QOL following attendance at a disease-specific residential camp [9].

The aim of the current study was to evaluate the shortterm impact of attendance at a residential IBD camp for children and adolescents on patients' disease-specific knowledge and QOL.

\section{Methods}

\section{Camp and Participants}

A 4-day residential camp for young people with IBD was held in Auckland, New Zealand, in January 2015. As described earlier, 44 children aged between 10 and 18 years of age attended the camp [8]. The children were supervised by 15 volunteer leaders, many of whom also had IBD. A medical team of 3 nurses and 3 gastroenterologists were present throughout the camp. The campers undertook a range of physical activities, including climbing, kayaking and archery. They could also participate in group activities, such as a mud slide and a campfire. The camp did not include specific IBD-related educational activities.

Participants were e-mailed questionnaires prior to, 1 month and 6 months after attendance at the camp following the obtainment of informed consent. Campers were also asked to provide details of background disease status and demographic information. The study was approved by the University of Otago Ethics Committee (Health).

\section{Questionnaires}

The Inflammatory Bowel Disease Knowledge Inventory Device (IBD-KID) was utilised to assess IBD-specific knowledge [7]. This 23 -item questionnaire was developed to assess disease-specific knowledge in children aged $>10$ years of age and has previously been validated in an Australasian setting [10]. The questionnaire was scored as the number of positive responses out of 23 (with 23 being the highest score and 0 being the lowest score).

IBD-specific QOL was assessed using the IMPACT-III questionnaire [11]. This tool features 35 questions and has been developed and validated for children aged 10-18 years. The tool com-

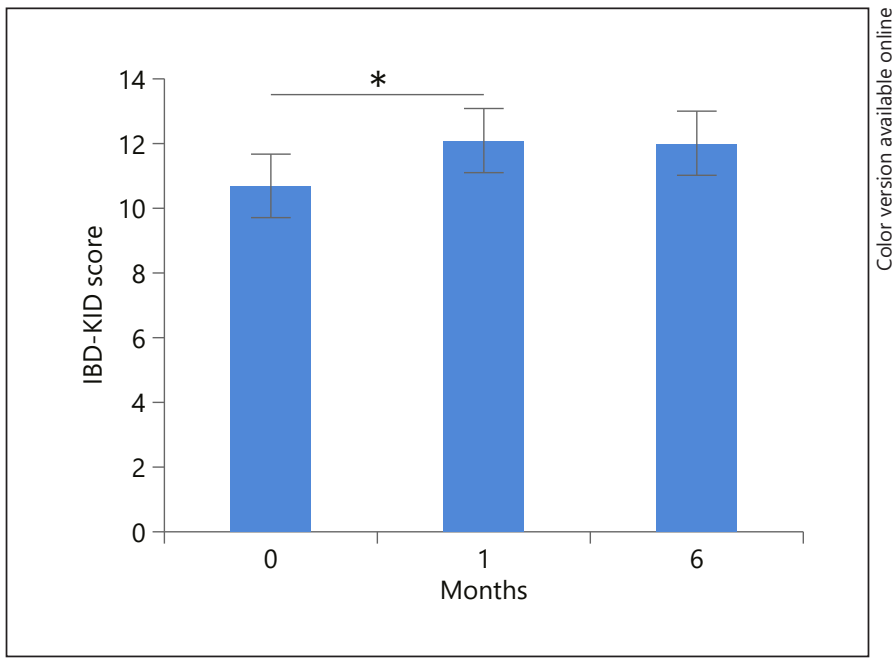

Fig. 1. IBD-specific knowledge (IBD-KID) assessment of campers before and after the New Zealand IBD camp. Knowledge scores were increased 1 month after camp compared to baseline. However, knowledge scores did not increase between 1 and 6 months after camp ${ }^{*}$ signifies $p$ value of 0.032 ).

prises 6 domains (bowel, systemic, emotional, social/functional, body image and treatments).

Disease activity at baseline was assessed by completion of a modified Harvey Bradshaw Index (HBI) [12]. A modified HBI of $\leq 4$ was indicative of remission.

Analysis

Results were analysed descriptively with means and standard deviations calculated. Comparisons were made using the paired Student's $t$ test, and statistical significance was accepted if the $p$ value was $<0.05$.

\section{Results}

\section{Participants}

Of the 44 children aged between 10 and 18 years who attended the camp, $39(88.6 \%)$ provided baseline responses, including the IBD-KID and IMPACT-III questionnaires. Twenty-one of the responders were male, 35 had Crohn's disease, and their median age was 14 years. Twenty-five of the 39 respondents were in clinical remission based on modified HBI scores.

\section{Baseline Knowledge and QOL Scores}

The respondents' initial IBD-KID scores ranged from 4 to 18: mean score was 10.7 (SD 4.1). IMPACT-III scores ranged from 81 to 170 , with a mean of 131.1 (SD 21.5). 
Follow-Up Knowledge and QOL Scores

One month following the camp, 31 IBD-KID and 30 IMPACT questionnaires were returned by camp participants. Six months following the camp, there were 28 and 25 IBD-KID and IMPACT questionnaires filled out by participants.

One month after the camp, $58.1 \%$ of campers had improved disease knowledge whilst $64.3 \%$ had scores above baseline at 6 months. Mean IBD-KID scores increased at 1 month to 12.1 (SD 3.4) ( $p=0.032$ compared to baseline) but did not increase further at 6 months (mean 12.0, SD $4.1 ; p=0.040$ compared to baseline, $p>0.05$ compared to 1-month scores; Fig. 1). Areas of poor knowledge were similar between groups (e.g., understanding of medication side effects, growth outcomes) at both time points. Similarly, areas of good knowledge were also similar. The number of "don't know" answers was greater at baseline; this was not significant (data not shown). However, there were overall improvements in specific areas of knowledge from baseline: these aspects were maintenance medication, post-surgical outcomes, impact of active disease on growth and corticosteroid side effects.

Although 50\% of children had improved QOL scores at 1 month, mean IMPACT-III scores were unchanged from baseline at both 1 month and 6 months $(p>0.05$, data not shown). Body image sub-scores were increased at 6 months $(p=0.015)$; however, other sub-scores did not change at either time point (data not shown).

\section{Discussion}

This study builds on previously published work by demonstrating further benefit derived by participants at an IBD residential camp. By using a validated measure of disease-specific knowledge (IBD-KID), attendees at New Zealand's first IBD camp were shown to have improvements in the short- and medium-term understanding of their chronic disease. However, average IMPACT-III scores did not change following the camp, although there was improvement seen in 1 sub-score.

The IBD-KID score was initially developed and validated in a Canadian setting [7]. A subsequent validation study confirmed the utility and reliability of IBD-KID in the Australasian setting [10]. A second larger study conducted in Australia showed that parental knowledge of IBD generally exceeded that of the patient/child [13]. Common areas of sub-optimal knowledge existed among paediatric Australian patients with IBD, and length of time since diagnosis positively correlated with disease-

Knowledge and Quality of Life after IBD specific knowledge. Similar strengths and weaknesses were evident in the current study population.

Improved disease understanding has been associated with better adherence to prescribed therapies. A previous study comprising adults with IBD examined the link between knowledge of disease and adherence to therapy and ability to cope with IBD [14]. Of interest, a positive association was established between disease-specific knowledge and adaptive coping strategies - these included greater active coping, planning, instrumental support and emotional support. However, the authors did not demonstrate a significant link between increased diseasespecific knowledge and adherence to prescribed therapies.

In the present study, attendance at a national IBD camp was associated with an improvement in participants' disease-specific knowledge 1 month after camp as measured by the IBD-KID tool. While no further improvement was noted between 1 and 6 months after the conclusion of the camp, there remained a significant increase in disease-specific knowledge at 6 months compared to baseline. The areas that appeared to improve related to aspects that children may have acquired during informal discussions with other campers (such as the role of maintenance medications). However, this improvement in knowledge regarding IBD amongst young people with the disease did not translate to objective improvement in QOL measurements at either 1 month or 6 months. Other implications of improved knowledge, such as adherence, were not assessed in the current study.

Previous work has demonstrated important psychosocial benefits of attendance at New Zealand's first IBD camp [8]. Greater than $80 \%$ of camp attendees reported an improvement in their confidence as it related to dealing with IBD and in their acceptance of having the chronic disease, whereas three-quarters of attendees listed an overall improvement in their QOL following attendance at the disease-specific camp. The vast majority of campers listed the opportunity to meet peers with IBD as the greatest benefit to attending the camp. These interactions with others, which likely provide informal discussions about aspects of IBD, may have contributed to improved disease-specific knowledge in the children in the current study.

Despite these subjective improvements, there was no change in QOL seen in these children using the IBD-specific and well-validated QOL tool IMPACT-III [11]. This may reflect in part that most of the children in the current study were in remission at the time of camp attendance. 
As disease activity influences health-related QOL, one would not expect subsequent improvements [15].

In contrast, health-related QOL did improve 2 weeks after a North American residential camp for children with IBD [16]. This evaluation involved 28 children with multiple assessments undertaken 2 weeks before and 2 weeks after an IBD camp. Particular improvements were seen in areas such as emotional function, school function and general psycho-social functioning. Children also reported improved subjective aspects such as greater personal strength related to their IBD after the camp. This report only examined QOL at 1 time point after camp and did not assess the duration of the effect.

This study utilised 2 validated patient-reported outcomes and provided promising results. These findings are limited by the small number of participants. Further work exploring these likely benefits from residential camps for young people with IBD needs to be evaluated in larger populations. In addition, this report focused on just 2 specific time points following the completion of the camp: the present results may have been augmented by evaluation at additional time points.

\section{Conclusion}

The psycho-social benefits of attendance at an IBD camp derived by its attendees have been demonstrated previously. This report suggests that improved diseasespecific knowledge can also follow from attendance at such a camp. While there was no significant increase in QOL following camp attendance based on the measures used, there was a trend towards improvement in certain domains. Further work delineating the benefits of diseasespecific camps for young patients with IBD are required.

\section{Acknowledgements}

Thank you to the children who participated in this study and to Crohn's and Colitis NZ for their support of this undertaking.

\section{Statement of Ethics}

The study was approved by the University of Otago Ethics Committee (Health) prior to commencement. Each subject (or their parent) provided written informed consent prior to involvement in the study. This work was conducted following the expected standards and guidelines throughout.

\section{Disclosure Statement}

None of the authors have any conflicts of interest to declare.

\section{Funding Sources}

There was no specific funding support.

\section{Author Contributions}

A.S.D. developed the concept for this study (with contributions from all authors) and guided the project throughout. R.N.L. assisted in the preparation of the study protocol, submission for ethics approval and preparation of the first manuscript draft. A.M. contributed to the development of the study protocol, data collection and data analysis. All authors contributed to the preparation of the manuscript, and all reviewed and approved the final version of the manuscript.

\section{References}

1 Benchimol EI, Fortinsky KJ, Gozdyra P, Van den Heuvel M, Van Limbergen J, Griffiths AM. Epidemiology of pediatric inflammatory bowel disease: a systematic review of international trends. Inflamm Bowel Dis. 2011 Jan; 17(1):423-39.

2 Su HY, Gupta V, Day AS, Gearry RB. Rising Incidence of Inflammatory Bowel Disease in Canterbury, New Zealand. Inflamm Bowel Dis. 2016 Sep;22(9):2238-44.

3 Lopez RN, Appleton L, Gearry RB, Day AS. Rising Incidence of Paediatric Inflammatory Bowel Disease in Canterbury, New Zealand,
1996-2015. J Pediatr Gastroenterol Nutr. 2018 Feb;66(2):e45-50.

4 Turnbull GK, Vallis TM. Quality of life in inflammatory bowel disease: the interaction of disease activity with psychosocial function. Am J Gastroenterol. 1995 Sep;90(9): 1450-4.

5 Kaplan SH, Greenfield S, Ware JE Jr. Assessing the effects of physician-patient interactions on the outcomes of chronic disease. Med Care. 1989 Mar;27(3 Suppl):S110-27.

6 Eaden JA, Abrams K, Mayberry JF. The Crohn's and Colitis Knowledge Score: a test for measuring patient knowledge in inflammatory bowel disease. Am J Gastroenterol. 1999 Dec;94(12):3560-6.

7 Haaland D, Day AS, Otley A. Development and validation of a pediatric IBD knowledge inventory device: the IBD-KID. J Pediatr Gastroenterol Nutr. 2014 Mar;58(3):313-9.

8 McCombie A, Gearry RB, Lopez RN, Lönnfors S, Day AS. Enhancements in Confidence, Acceptance, and Friendship at a Summer Camp for Children with Inflammatory Bowel Disease. J Pediatr Gastroenterol Nutr. 2017 Sep;65(3):311-3. 
9 Shepanski MA, Hurd LB, Culton K, Markowitz JE, Mamula P, Baldassano RN. Healthrelated quality of life improves in children and adolescents with inflammatory bowel disease after attending a camp sponsored by the Crohn's and Colitis Foundation of America. Inflamm Bowel Dis. 2005 Feb;11(2):164-70.

10 Day AS, Lemberg DA, Nichol A, Clarkson C, Otley AR. Generalisability of the inflammatory bowel disease knowledge inventory device to assess disease-related knowledge in Australian children. J Paediatr Child Health. 2014 Aug;50(8):591-5.

11 Otley A, Smith C, Nicholas D, Munk M, Avolio J, Sherman PM, et al. The IMPACT questionnaire: a valid measure of health-related quality of life in pediatric inflammatory bow- el disease. J Pediatr Gastroenterol Nutr. 2002 Oct;35(4):557-63.

12 Zittan E, Kabakchiev B, Kelly OB, Milgrom R, Nguyen GC, Croitoru K, et al. Development of the Harvey-Bradshaw Index-pro (HBIPRO) Score to Assess Endoscopic Disease Activity in Crohn's Disease. J Crohns Colitis. 2017 May; 11(5):543-8.

13 Day AS, Mylvaganam G, Shalloo N, Clarkson C, Leach ST, Lemberg DA. Assessment of disease-specific knowledge in Australian children with inflammatory bowel disease and their parents. J Paediatr Child Health. 2017 Aug;53(8):778-81.

14 Moradkhani A, Kerwin L, Dudley-Brown S, Tabibian JH. Disease-specific knowledge, coping, and adherence in patients with in- flammatory bowel disease. Dig Dis Sci. 2011 Oct;56(10):2972-7.

15 Otley AR, Griffiths AM, Hale S, Kugathasan S, Pfefferkorn M, Mezoff A, et al.; Pediatric IBD Collaborative Research Group. Healthrelated quality of life in the first year after a diagnosis of pediatric inflammatory bowel disease. Inflamm Bowel Dis. 2006 Aug;12(8): 684-91.

16 Lawton R, Rothbaum R, LaRose-Wicks M, Weissberg-Benchell J, Victorson D, Washburn J. Conceptualizing summer camp as a psychosocial intervention: significant improvement in emotional function and healthrelated quality of life after participation in a summer camp for youth with IBD. J Pediatr Gastroenterol Nutr. 2016 Oct;63(Suppl2):S81. 